\title{
三光束激光熔覆工艺参数对熔覆层几何特征影响 ${ }^{*}$
}

\author{
刘凡吉绍山傅戈雁石世宏 \\ (苏州大学机电工程学院 苏州 215021)
}

\begin{abstract}
摘要: 采用 “三光束光内同轴送丝” 激光熔覆新方法研究可以获得表面质量较高单道熔覆层的工艺参数组合方案。建立三光 束激光熔覆工艺参数与熔覆层表面形貌关系以及几何特征变化规律的数学模型, 通过激光提供的单位能量密度 $E_{1}$ 与丝材所 需的能量密度 $E_{2}$ 之间比值关系判断熔覆层表面形貌状态，工艺参数和熔覆层几何特征关系的数学模型预测熔覆层几何特征 变化规律, 并采用单因素试验法进行验证。研究结果如下: $E_{1} / E_{2}<1$, 熔池能量 “不足”, $1.1 \geqslant E_{1} / E_{2} \geqslant 1.0$, 熔池能量处于 “不 足” 到 “充足” 过渡阶段, 以及 $E_{1} / E_{2}>1.6$, 熔池能量 “过剩” , 这三种情况形成的熔覆层表面质量较差; 只有当 $1.5 \geqslant$ $E_{1} / E_{2}>1.1$, 熔池能量 “充足” 能够充分熔化进入熔池的丝材, 且丝材能够以连续平稳的 “搭桥过渡” 方式熔入熔池, 熔覆层 表面连续光滑, 质量较高; 熔覆层几何特征变化规律: 数学模型预测值变化趋势与试验值基本吻合, 离焦量减小, 熔覆层宽 度 $W$ 减小, 高度 $H$ 增大, 宽高比 $a$ 减小; 激光功率增大, 熔覆层宽度 $W$ 增大, 高度 $H$ 减小, 宽高比 $a$ 增大。扫描速度增大, 熔覆层宽度 $W$ 减少, 高度 $H$ 减少, 宽高比 $a$ 略有增加。送丝速度增大, 熔覆层宽度 $W$ 和高度 $H$ 都会增大, 宽高比 $a$ 减小。 综上采用 “三光束光内同轴送丝” 新方法对不锈钢 304 材料的丝材和基材进行激光熔覆试验, 工艺参数组合方案满足 $1.5 \geqslant$ $E_{1} / E_{2}>1.1$, 可以制备出表面形貌佳熔覆层。
\end{abstract}

关键词: 激光熔覆; 三光束; 光内送丝; 工艺参数; 几何特征

中图分类号: TN249; TF124

\section{The Influence of Process Parameters on Geometry Characteristics by Three Beams Laser Cladding}

\author{
LIU Fan JI Shaoshan FU Geyan SHI Shihong \\ (College of Mechanical and Electrical Engineering, Soochow University, Suzhou 215021)
}

\begin{abstract}
In order to obtain better combination scheme of process parameters of single cladding layer with high surface quality, a new forming method of "coaxial wire feeding inside three beams" is used. Mathematical model of relationship between process parameters of layer cladding and the surface morphology is established, between process parameters and geometric characteristics. The surface morphology state of cladding layer is judged by the ratio between the unit energy density provided by the laser $E_{1}$ and the required energy density of wire $E_{2}$.The mathematical model of the relationship between the process parameters and the geometric characteristics of cladding layer is used to predict the variation law of the geometric characteristics, and the single factor test method is used to make experimental verification. The experienced state of cladding layer surfacemorphology is that: $E_{1} / E_{2}<1$, the molten pool energy is "insufficient”, $1.1 \geqslant E_{1} / E_{2} \geqslant 1.0$, the energy of molten pool is in the transition stage from "insufficient” to "sufficient”, $E_{1} / E_{2}>1.6$, the energy of the molten pool is "excess", the surface quality of the coating formed in these three cases is poor. Only when $1.5 \geqslant E_{1} / E_{2}>1.1$, the "sufficient" energy of the molten pool can fully melt the wire, which can be melted into the molten pool in a continuous and stable way, the surface of cladding layer is continuously smooth and the quality is high. The variation law of cladding layer geometric characteristics: the predicted value of the mathematical model is in good agreement with the experimental value, the defocusing amount decreases, the width $W$ decreases, the height $H$ increases, and the ratio of width to height $a$ decreases. Laser power increases, the width $W$ increases, the height $H$ decreases, and the ratio of width to height a increases. Scanning speed increases, the width $W$ decreases, the height $H$ decreases, and the ratio of width to height a increases slightly. Wire feeding speed increases, the width $W$ and height $H$ increases, and the ratio of width to height adecreases. Conclusion is that: make laser cladding test of stainless steel 304 wire and substrate by using new forming method of "coaxial wire feeding inside three beams”, selecting reasonable process parameters scheme, which meet $1.5 \geqslant E_{1} / E_{2}>1.1$, better surface morphology can be achieved.
\end{abstract}

* 国家重点研发专项(2017YFB1103601)、国家自然科学基金(61903268, 51701134)、江苏省自然科学基金(BK20190823)和中国博士后基金 (2019M661921)资助项目。20191022 收到初稿, 20200405 收到修改稿 
Key words: laser cladding; three beams; wire feeding inside beams; process parameter; geometric characteristics

\section{0 前言}

激光熔覆技术是采用高能量激光束作为热源, 在基材表面通过添加金属粉末或丝材实现改变表 面性能的技术 ${ }^{[1]}$ 。国内外采用熔覆方法主要是送粉 法, 其利用率低、易污染环境以及部分未熔颗粒黏 附在熔覆层外表面, 影响成形表面质量 ${ }^{[2-6]}$ 。而激 光送丝熔覆技术具有：材料利用率高达 $100 \%$, 节 能环保, 成本低, 丝材输送更易精确控制, 因此具 有很好的发展前景 ${ }^{[2-5]}$ 。而目前采用的 “侧向送丝 法”, 其问题有 ${ }^{[2-6]}$ : (1) 光斑和丝材相对位置不同, 进行不同方向扫描时呈现熔覆层形貌与质量各向 异性; (2) 离焦量变化导致光斑和丝材位置关系变 化, 无法实现精确光、丝耦合; (3) 丝材相对激光 束倾斜布置, 受热不均匀。这些缺陷制约了送丝激 光熔覆技术推广应用。傅戈雁课题组提出了 “三光 束光内同轴送丝” 激光熔覆方法, 原理如图 1 所 示: 激光束通过三棱镜分成周向均匀三光束, 再通 过聚焦镜反射并聚焦到基材上，在三光束中空区 域, 丝材通过导丝管、导丝嘴通道, 最终与光束同 轴进入到熔池区域。其特点 ${ }^{[7-10]}$ 为：(1) 丝材与三 个光束同轴布置, 实现了光、丝精确耦合, 且丝材 受热均匀, 有助于提高熔覆层质量; (2) 避免光丝 干涉, 激光能量利用率高。

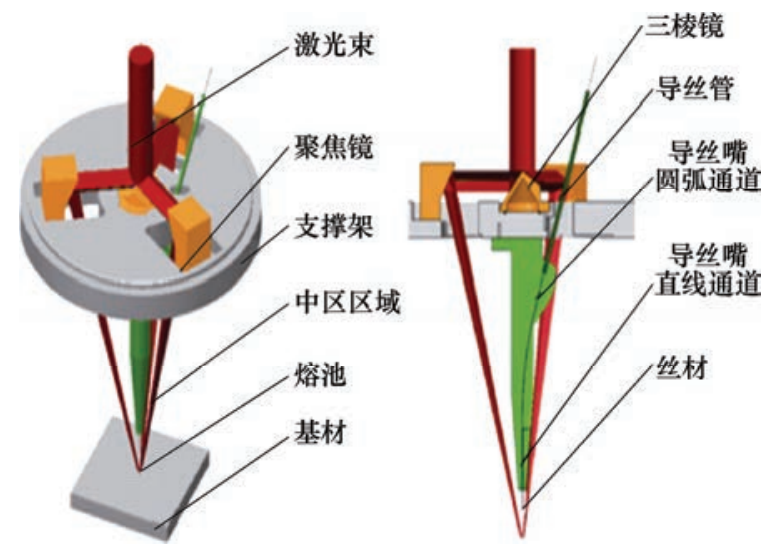

图 1 三光束光内同轴送丝原理图

影响激光熔覆质量关键影响因素是激光熔覆工 艺参数, 国内外学者投入了大量精力研究激光熔覆 工艺对熔覆层质量影响, 取得一定成果。李凯斌

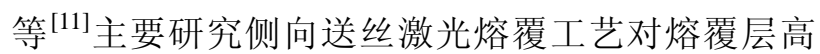
度和宽度影响程度, 结果表明: 激光功率对熔覆层 宽度影响最大, 送丝速度对熔覆层高度影响最大。 罗园青 ${ }^{[12]}$ 采用单因素工艺试验研究侧向送丝激光
熔覆工艺对单道熔覆层表面质量影响, 结果表明, 丝材位于熔池中心并前向送丝，最佳工艺参数区间: 激光功率 $P=2000 \sim 2800 \mathrm{~W}$, 扫描速度 $V_{s}=0.27 \sim$ $0.54 \mathrm{~m} / \mathrm{min}$, 送丝速度 $V_{f}=2.0 \sim 3.2 \mathrm{~m} / \mathrm{min}$, 可以获 得较好熔覆层表面质量, 有效保证零件的成形精度。 MOK 等 ${ }^{[13]}$ 研究侧向送丝激光熔覆工艺参数对熔覆 层宽度和高度影响, 结果表明: 送丝角度 $45^{\circ}$ 最有 利于单道熔覆, 对熔覆层宽度影响最大的是激光功 率, 对熔覆层高度影响最大的是扫描速度。ABIOYE 等 ${ }^{[14]}$ 采用侧向送丝激光熔覆方法对铬镍铁合金 625 进行工艺参数的研究, 结果表明: 影响熔覆层几何 特征主要因素有单位熔覆层质量和线能量密度, 送 丝速度的增大, 接触角增大, 稀释率减小; 扫描速 度增大时, 稀释率增大, 接触角减小; 稀释率和宽 高比与激光功率成正比, 接触角与之成反比。LIU 等 ${ }^{[15]}$ 主要研究侧向送热丝激光熔覆工艺对熔覆层 表面质量以及稀释率影响, 结果表明: 激光功率范 围为 2 4 kW，扫描速度范围为 5 15 mm/s, 送丝 速度为 $90 \mathrm{~mm} / \mathrm{s}$, 激光光斑尺寸范围为 $2 \sim 6 \mathrm{~mm}$, 当光斑尺寸为 $2 \mathrm{~mm}$ 时, 熔覆层表面成球状且熔深 很大, 熔覆层表面质量较差; 当光斑尺寸为 $6 \mathrm{~mm}$ 时, 可以形成较好的熔覆层表面质量, 激光功率越大, 稀释率越大，扫描速度在 5 $10 \mathrm{~mm} / \mathrm{s}$ 区间，扫描速 度越大, 稀释率增大，扫描速度在 $10 \sim 15 \mathrm{~mm} / \mathrm{s}$, 扫描速度增大，稀释率反而减小。BARROI 等 ${ }^{[16]}$ 采 用激光横向扫描侧向送丝技术研究扫描速度对熔覆 层高度的波动影响, 以及激光功率对熔覆层高度和 宽度的影响分析, 结果表明: 激光功率为 $640 \mathrm{~W}$, 光斑沿熔覆层方向尺寸为 $2.9 \mathrm{~mm}$, 光斑沿熔覆层垂 直方向的尺寸为 $4 \mathrm{~mm}$ 时, 扫描速度为 $2.2 \mathrm{~mm} / \mathrm{s}$ 时, 熔覆层高度尺寸波动最小; 激光功率区间为 475 $805 \mathrm{~W}$, 光斑沿熔覆层方向尺寸为 $2.9 \mathrm{~mm}$, 光斑沿 熔覆层垂直方向的尺寸为 $4 \mathrm{~mm}$ 时, 扫描速度为 $2.2 \mathrm{~mm} / \mathrm{s}$ 时, 激光功率增大, 熔池面积增加, 熔深 增加, 熔覆层宽度增大, 高度减小。WEN 等 ${ }^{[17]}$ 采 用高速相机研究侧向送热丝激光熔覆成形过程中丝 材变化特性, 结果表明: 丝材在激光熔覆过程中主 要经历三种状态: 熔化转换状态, 连续转换状态, 以及丝材顶撞状态，只有当丝材出现连续转换状态 可以获得质量较高的熔覆层。

熔覆层表面形貌和几何特征(熔覆层的宽度、高 度、宽高比)是衡量熔覆层质量的重要指标, 上述研 究人员主要针对侧向送丝法激光熔覆工艺对熔覆质 量影响研究，而对 “三光束光内 同轴送丝” 激光熔 
覆的新方法研究甚少, 本文采用 “三光束光内同轴 送丝” 激光熔覆装置, 采用工艺参数组合的数学模 型和单因素试验法重点研究熔覆层表面形貌状态和 几何特征变化规律。

\section{1 试验方案}

\section{1 试验装置}

三光束光内同轴送丝熔覆装置如图 2 所示 ${ }^{[18]}$ 。 IPG 品牌 $2 \mathrm{~kW}$ 光纤激光器和准直镜通过接口与喷头 连接, 机器人通过支架与喷头连接, 激光束通过喷头 内部的三棱镜和聚焦镜实现分光和聚焦, 丝材通过送 丝软管输送到喷头内部通道, 并从导丝嘴流出, 实现 光、丝的精确耦合, 保护气体通过气体输入口以及喷 头内部通道输送到熔覆层表面, 防止熔覆层氧化。

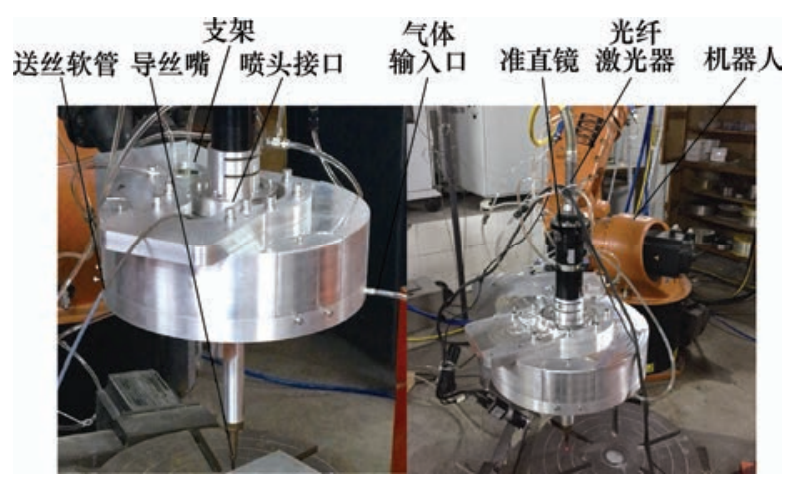

图 2 三光束熔覆装置图

\section{2 试验材料}

基体材料：选用尺寸 $150 \mathrm{~mm} \times 150 \mathrm{~mm} \times 10 \mathrm{~mm}$ (长 $\times$ 宽 $\times$ 高) 304 不锈钢。熔覆前基体用酒精/丙酮去 除油污，烘干备用。

熔覆材料: 选用 304 不锈钢丝材, 直径为 0.8 $\mathrm{mm}$ ，其成分如表 1 所示。

表 1 基材和丝材化学成分占比

\begin{tabular}{cc||cc}
\hline 成分 & 质量分数 & 成分 & 质量分数 \\
\hline $\mathrm{C}$ & $\leqslant 0.07$ & $\mathrm{P}$ & $\leqslant 0.035$ \\
$\mathrm{Si}$ & $\leqslant 1.0$ & $\mathrm{Ni}$ & $8.0 \sim 11.0$ \\
$\mathrm{Mn}$ & $\leqslant 2.0$ & $\mathrm{~S}$ & $\leqslant 0.03$ \\
$\mathrm{Cr}$ & $17.0 \sim 19.0$ & $\mathrm{Fe}$ & 其他 \\
\hline
\end{tabular}

\section{3 试验方法}

采用工艺参数组合的数学模型和单因素试验法 逐个研究离焦量、激光功率、扫描速度以及送丝速 度对单道熔覆层表面形貌状态影响和几何特征(宽 度、高度、宽高比)的变化规律, 并得到获得较好熔 覆层表面质量的工艺参数组合方案。测量熔覆层宽 度和高度并计算宽高比，测量方式如图 3 所示。

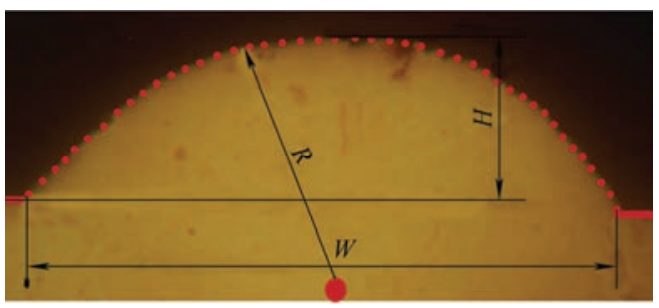

图 3 熔覆层宽度、高度测量方法

\section{2 试验结果与讨论}

\section{1 离焦量}

\subsection{1 试验结果}

离焦量是指焦点相对基材表面的坚直方向的距 离, 焦点在基材表面时离焦量为零, 焦点在基材表 面上方称正离焦，反之称负离焦。三光束光内同轴 送丝激光熔覆装置三种离焦情况如图 4 所示。

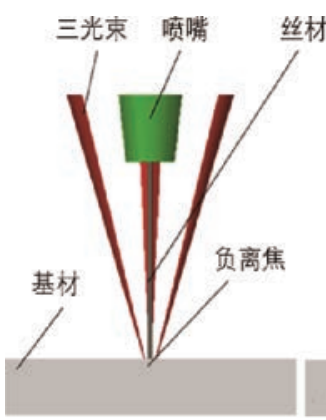

(a) 负离焦位置

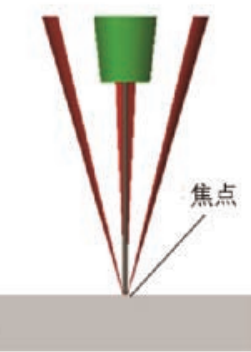

(b) 焦点位置

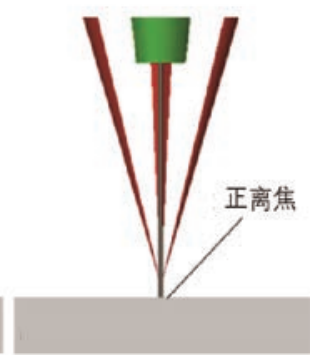

(c) 正离焦位置

图 4 不同离焦量熔覆图

表 2 为激光功率 $1.3 \mathrm{~kW}$ ，送丝速度 $20.5 \mathrm{~mm} / \mathrm{s}$, 扫描速度 $6 \mathrm{~mm} / \mathrm{s}$, 离焦量-4 1 $\mathrm{mm}$ 区间单道熔覆 层表面形貌经历五种状态: (1) 离焦量为 $-4 \mathrm{~mm}$ 时, 熔覆层表面呈 “残缺” 状，局部有 “粘丝” 现象; (2) 离焦量为 $-3 \mathrm{~mm}$ 时, 可以形成连续熔覆层, 但熔 覆层局部区域有 “凹陷” 状; (3) 离焦量为 $-2.5 \mathrm{~mm}$
和-2 mm 时, 熔覆层表面连续光滑且均匀; (4) 离焦 量-1 mm 时, 熔覆层呈 “波浪” 状; (5) 离焦量为 $-0.5 \mathrm{~mm} 、+0.5 \mathrm{~mm}$ 和 $+1 \mathrm{~mm}$ 时, 熔覆层表面呈不 连续的 “熔滴” 状。离焦量 $-3 \sim-1 \mathrm{~mm}$ 区间可以形 成整道熔覆层, $-2.5 \mathrm{~mm}$ 和 $-2 \mathrm{~mm}$ 可以获得较好熔 覆层表面质量。其几何特征参数：如图 6 所示，离 
焦量减小, 熔覆层宽度 $W$ 减小, 高度 $H$ 增加, 宽高 比 $a$ 减小。

表 2 不同离焦量单道熔覆层表面形貌

\begin{tabular}{cccc}
\hline \multirow{2}{*}{ 试样 } & 离焦量/mm & \multicolumn{2}{c}{ 表面形貌 } \\
\cline { 3 - 4 } & & 图片 & 状态 \\
\hline 1 & -4.0 & (1) \\
2 & -3.0 & (2) \\
3 & -2.5 & (3) \\
4 & -2.0 & \\
5 & -1.0 & \\
6 & -0.5 & & (3) \\
7 & 0.5 & & (5) \\
8 & 1.0 & & (5) \\
\hline
\end{tabular}

\subsection{2 表面形貌分析}

(1) 表面形貌数学模型。

激光熔覆过程中熔覆材料和基体受到一定的热 量且达到熔点后, 熔覆层才能够熔化并形成形状规 则的熔覆层, 丝材的加热过程主要是从室温 $T_{0}$ 加热 到熔点 $T_{m}$, 最后达到最终稳态温度 $T_{f}$ 。

三光束同轴送丝激光熔覆过程中, 激光加热时 间短, 熔覆过程极其复杂, 需要进行如下简化计算:

(1) 熔覆材料以及基材在固态和熔化状态下, 除密度变 化以外, 其他热物理参数保持不变; (2) 丝材与基材 材料质量均匀, 各向同性; (3) 丝材始终垂直于基材 表面, 丝材速度为恒速, 其初始位置丝材底面与基材 完全接触; (4) 热量损耗主要考虑丝材的热辐射和热 对流, 忽略保护气体对热量损耗的影响; (5) 忽略基 体吸热影响, 将基体吸收能量进而升温的过程作为绝 热过程; (6) 照射到基材上的三个光斑之间距离非常 小, 将三个光斑简化为沿着其外径相切直径为 $d_{2}$ 单个 圆光斑, 本文采用其理论尺寸, 见文献[18]; (7) 在负 离焦状态, 光斑全部照射到基材, 丝材所需能量主要 由基体熔池提供; (8) 在正离焦状态, 光斑全部照射 到丝材, 即激光所有能量都作用在丝材上。

设激光输出功率 $P$ 、光束通过喷头分光镜和聚 焦镜, 其总吸收率为 $\eta_{1}$ 为 $2 \%{ }^{[19]}$, 基材的吸收率 $\eta_{2}$ 区间为 $0.16 \sim 0.85^{[20-21]}$, 吸收率选择 0.24 , 熔覆层 总长度为 $L$, 扫描速度为 $V_{s}$, 则作用在丝材的总能 量为

$$
Q_{1}=\left(1-\eta_{1}\right) \eta_{2} P\left(\frac{L}{V_{S}}\right)
$$

设丝材的半径 $r$, 送丝速度 $V_{f}$, 丝材在室温下 密度为 $\rho_{0}$, 熔化潜热 $L_{m}$, 丝材由室温 $T_{0}$ 加热到熔 点 $T_{m}$, 丝材熔化所需要吸收能量

$$
Q_{2}=\pi r^{2} V_{f}\left(\frac{L}{V_{S}}\right) \rho_{0}\left(C\left(T_{m}-T_{0}\right)+L_{m}\right)
$$

丝材受热熔化时也会产生向外辐射能量和对流 导热等现象, 丝材表面积 $S_{w}$, 丝材向外辐射能量的

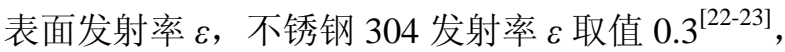
Stefan-Boltzmann 常数 $\sigma$, 热对流系数 $h$, 令 $Q_{e}$ 和 $Q_{c}$ 分别表示丝材向外辐射和对流导热所损耗的能 量, 则根据传热学公式可得到

$$
\begin{gathered}
Q_{e}=S_{W} \varepsilon \sigma\left(T_{m}^{4}-T_{0}^{4}\right)\left(\frac{L}{V_{S}}\right)= \\
2 \pi r V_{f} \varepsilon \sigma\left(\frac{L}{V_{S}}\right)\left(T_{m}^{4}-T_{0}^{4}\right)\left(\frac{L}{V_{S}}\right)= \\
2 \pi r V_{f} \varepsilon \sigma\left(T_{m}^{4}-T_{0}^{4}\right)\left(\frac{L}{V_{S}}\right)^{2} \\
Q_{c}=h S_{w}\left(T_{m}-T_{0}\right)\left(\frac{L}{V_{S}}\right)= \\
2 h \pi r V_{f}\left(\frac{L}{V_{S}}\right)\left(T_{m}-T_{0}\right)\left(\frac{L}{V_{S}}\right)= \\
2 h \pi r V_{f}\left(T_{m}-T_{0}\right)\left(\frac{L}{V_{S}}\right)^{2}
\end{gathered}
$$

考虑辐射能量计算相对比较复杂, 为了简化计算, 将 辐射和对流进行整合计算 ${ }^{[23]}$, 其热对流系数 $h$ 公式为

$$
h=2.4 \times 10^{-3} \varepsilon T_{m}^{1.61}
$$

将上述辐射和对流所损耗的能量整合为

$$
\begin{gathered}
Q_{3}=2.4 \times 10^{-3} \varepsilon T_{m}^{1.61} 2 \pi r V_{f}\left(T_{m}-T_{0}\right)\left(\frac{L}{V_{S}}\right)^{2}= \\
4.8 \times 10^{-3} \varepsilon T_{m}^{1.61} \pi r V_{f}\left(T_{m}-T_{0}\right)\left(\frac{L}{V_{S}}\right)^{2}
\end{gathered}
$$

则作用在丝材有效总能量为

$$
\begin{gathered}
Q=Q_{1}-Q_{3}=\left(1-\eta_{1}\right) \eta_{2} P\left(\frac{L}{V_{s}}\right)-4.8 \times \\
10^{-3} \varepsilon T_{m}^{1.61} \pi r V_{f}\left(T_{m}-T_{0}\right)\left(\frac{L}{V_{S}}\right)^{2}
\end{gathered}
$$

考虑离焦量变化会产生光斑尺寸相应变化, 需 要引入激光单位能量密度才能够准确评估提供给丝 材的总能量以及丝材所需能量的关系, 负离焦状态, 作用在基体表面单位能量密度为

$$
\begin{gathered}
E_{1}=\frac{Q_{1}-Q_{3}}{d_{2} L}= \\
{\left[\left(1-\eta_{1}\right) \eta_{2} P\left(\frac{L}{V_{S}}\right)-\right.}
\end{gathered}
$$

$\left.4.8 \times 10^{-3} \varepsilon T_{m}^{1.61} \pi r V_{f}\left(T_{m}-T_{0}\right)\left(\frac{L}{V_{S}}\right)^{2}\right] / d_{2} L$

由假设条件(3)与(7), 丝材底面与基体完全接触, 
负离焦状态, 则丝材所需单位能量密度为

$$
\begin{gathered}
E_{2}=\frac{Q_{2}}{2 r L}=\frac{\pi r^{2} V_{f}\left(\frac{L}{V_{S}}\right) \rho_{0}\left(C\left(T_{m}-T_{0}\right)+L_{m}\right)}{2 r L} \\
E_{3}=\frac{Q_{1}-Q_{3}}{2 \pi r V_{f}\left(\frac{L}{V_{S}}\right)}=\frac{\left(1-\eta_{1}\right)\left(1-\gamma_{o}\right)}{2 \pi r V_{f}\left(\frac{L}{V_{S}}\right)}= \\
\frac{E_{4}=\frac{Q_{2}}{r^{2} V_{f}\left(\frac{L}{V_{S}}\right) \rho_{0}\left(C\left(T_{m}-T_{0}\right)+L_{m}\right)}}{2 \pi r V_{f}\left(\frac{L}{V_{S}}\right)}
\end{gathered}
$$$$
E_{3}=\frac{Q_{1}-Q_{3}}{2 \pi r V_{f}\left(\frac{L}{V_{S}}\right)}=\frac{\left(1-\eta_{1}\right)\left(1-\gamma_{\omega}\right) P\left(\frac{L}{V_{S}}\right)-4.8 \times 10^{-3} \varepsilon T_{m}^{1.61} \pi r V_{f}\left(T_{m}-T_{0}\right)\left(\frac{L}{V_{S}}\right)^{2}}{2 \pi r V_{f}\left(\frac{L}{V_{S}}\right)}
$$
材总单位能量密度以及丝材所需单位能量密度分 别为

为 $6 \mathrm{~mm} / \mathrm{s}$, 熔覆层长度 $L$ 为 $60 \mathrm{~mm}$, 采用数学模型 式(8) (11)进行计算, 得出负离焦时 $E_{1} 、 E_{2}$ 以及 $E_{1} / E_{2}$ 比值和正离焦时 $E_{3} 、 E_{4}$ 以及 $E_{3} / E_{4}$ 比值, 其计 算结果如表 4 所示。

根据丝材熔化状态变化, 可以得出: $E_{1} \geqslant E_{2}$, 即 $E_{1} / E_{2} \geqslant 1$, 负离焦状态, 作用在丝材的总单位 能量密度 $E_{1}$ 大于丝材所需单位能量密度 $E_{2}$, 才可以 使得丝材处于熔化状态; $E_{3} \geqslant E_{4}$, 即 $E_{3} / E_{4} \geqslant 1$, 正离焦状态, 同样也可以熔化丝材。

本文采用文献[24-26]中的 304 不锈钢材料属性 参数, 如表 3 所示。熔覆工艺参数选择激光功率 $P$ 为 $1.3 \mathrm{~kW}$, 送丝速度 $V_{f}$ 为 $20.5 \mathrm{~mm} / \mathrm{s}$, 扫描速度 $V_{s}$

表 4 不同离焦量单位能量密度值

\begin{tabular}{cccccccc}
\hline 离焦量 $/ \mathrm{mm}$ & -4.0 & -3.0 & -2.5 & -2.0 & -1.0 & -0.5 \\
\hline$E_{2}\left(E_{4}\right) /\left(\mathrm{J} / \mathrm{m}^{2}\right)$ & $1.7 \times 10^{7}$ & $1.7 \times 10^{7}$ & $1.7 \times 10^{7}$ & $1.7 \times 10^{7}$ & $1.7 \times 10^{7}$ & $1.7 \times 10^{7}$ & 0.5 \\
$E_{1}\left(E_{3}\right) /\left(\mathrm{J} / \mathrm{m}^{2}\right)$ & $1.4 \times 10^{7}$ & $1.9 \times 10^{7}$ & $2.2 \times 10^{7}$ & $2.7 \times 10^{7}$ & $5.7 \times 10^{7}$ & $11.4 \times 10^{7}$ & $2.1 \times 10^{7}$ \\
$E_{1} / E_{2}\left(E_{3} / E_{4}\right)$ & 0.8 & 1.1 & 1.3 & 1.6 & 3.4 & $0.2 \times 10^{7}$ & $2.1 \times 10^{7}$ \\
\hline
\end{tabular}

(2) 表面形貌分析。

由图 5 和表 4 可知, 负离焦时, 离焦量越大, 光斑的外径就越大, 激光提供单位能量密度 $E_{1}$ 就越 低, 即熔池能量低, 而离焦量越小, 则得到相反的 结果。离焦量 $-4.0 \mathrm{~mm}$ 时, 提供单位能量密度 $E_{1}$ 低 于丝材所需的激光能量密度 $E_{2}$, 其 $E_{1} / E_{2}$ 为 0.8 , 即 熔池能量 “不足” 无法充分熔化进入熔池丝材, 刚 性不足的丝材顶到基材, 受到基材反作用力会偏离 光斑中心, 出现时而进入和脱离熔池, 光丝耦合不 稳定, 部分丝材无法充分熔化并粘附在熔覆层表面, 出现形貌(1)的情况: 熔覆层表面呈 “残缺” 状, 局 部有 “粘丝” 现象, 不能形成连续光滑平整的熔覆 层; 离焦量 $-3.0 \mathrm{~mm}$ 时, $E_{1} / E_{2}$ 比值为 1.1 , 熔池的 能量处于刚好熔化丝材临界状态, 虽然可以形成整 道熔覆层, 但丝材依然会轻微顶到基材, 搅动熔池, 导致熔覆层表面还不够饱满, 出现形貌(2)的情况: 可以形成整道熔覆层, 但局部区域有小的 “凹陷”
状; 离焦量 $-2.5 \mathrm{~mm}$ 和 $-2.0 \mathrm{~mm}$ 时, 提供单位能量 密度 $E_{1}$ 明显提高, $E_{1} / E_{2}$ 比值达到 $1.3 \sim 1.6$, 说明熔 池能量 “充足” 可以充分熔化进入熔池的丝材, 熔 池对流充分，由于熔池表面张力的作用下，丝材以 连续平稳的 “搭桥过渡” 方式 ${ }^{[4]}$ 熔入熔池, 获得了 熔覆层表面连续光滑且均匀的形貌(3), 表面质量较 高。离焦量 $-1 \mathrm{~mm}$ 时, 激光单位功率密度 $E_{1}$ 更高, $E_{1} / E_{2}$ 比值达到 3.4, 是离焦量 $-2.0 \mathrm{~mm}$ 的 2 倍多, 说明熔池的能量略显 “过剩” , 熔池的对流非常充 分, 丝材未进入熔池前就已经开始熔化, 由于丝材 刚性弱, 其末端熔滴会左右偏摆, 光斑尺寸变小导 致熔池变小, 被摚动的溶液更易溢出熔池两侧, 产 生形貌(4)现象: 熔覆层呈 “波浪” 状。离焦量-0.5 $\mathrm{mm}$ 时, 激光单位能量密度 $E_{1}$ 极高, $E_{1} / E_{2}$ 比值达 到 6.7, 即提供单位能量密度 $E_{1}$ 远远大于丝材熔化 所需要的能量密度 $E_{2}$, 熔池能量 “过剩” 导致丝 材未进入熔池前就提前熔化, 熔覆层表面呈不连续 
的 “熔滴” 状 ${ }^{[4]}(5)$ 。正离焦 $0.5 \mathrm{~mm} 、 1.0 \mathrm{~mm}$, 丝材 进入熔池前, 首先通过焦点位置, 光斑几乎全部照 射到丝材表面, 导致单位能量密度 $E_{3}$ 极高, $E_{3} / E_{4}$ 比值达到了 10.5, 作用在丝材上的能量严重“过剩”
导致在熔池的上空形成熔滴, 重力和保护气体吹力 作用下进入熔池，形成不连续的 “熔滴” 状(5)熔覆 层 $^{[4]}$ 。所以要获得较好表面形貌质量需要选择合适 的负离焦量。

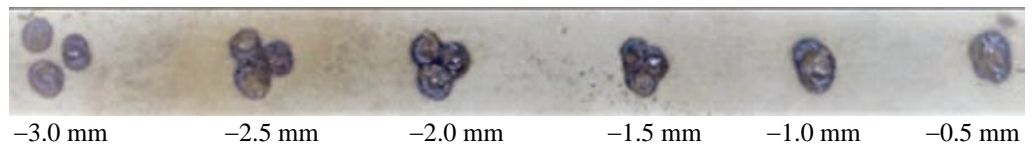

图 5 不同离焦量的三光束光斑烧蚀图

\subsection{3 几何特征分析}

(1) 几何特征数学模型。

温度达到稳态温度 $T_{f}$ 时, 根据能量守恒定律可知

$$
E_{1}=E_{2}
$$

由式(8)、(9)得出

$$
\frac{\pi r^{2} V_{f}\left(\frac{L}{V_{S}}\right) \rho_{0}\left(C\left(T_{f}-T_{0}\right)+\mathrm{L}_{m}\right)}{2 r L}=\frac{1}{d_{2} L} \times
$$

$\left(\left(1-\eta_{1}\right) \eta_{2} P\left(\frac{L}{V_{S}}\right)-4.8 \times 10^{-3} \varepsilon T_{f}^{1.61} \pi r V_{f}\left(T_{f}-T_{0}\right)\left(\frac{L}{V_{S}}\right)^{2}\right)$

再根据质量守恒定律

$$
S V_{s} t=\pi r^{2} V_{f} t
$$

式中, $S$ 为熔覆层横截面积, 由式(13)、(14)求解出

$$
\begin{gathered}
S=\frac{1}{V_{S} d_{2} \rho_{0}\left(C\left(T_{f}-T_{0}\right)+L_{m}\right)} \times \\
\left(2 r\left(\left(1-\eta_{1}\right) \eta_{2} P-4.8 \times 10^{-3} \varepsilon T_{f}^{1.61} \pi r V_{f}\left(T_{f}-T_{0}\right)\left(\frac{L}{V_{S}}\right)\right)\right)
\end{gathered}
$$

通过观察表面形貌质量较高的熔覆层, 其横截 面的外轮廓近似认为半径为 $R$ 的半圆弧形状, 如图 3 所示, 熔覆层的几何特征主要由宽度 $W$ 、高度 $H$ 、 圆弧半径 $R$, 其圆弧中心位于熔覆层下方, 则它们 关系式为

$$
\begin{gathered}
S=R^{2} \arcsin \frac{W}{2 R}-\frac{1}{2} W \sqrt{R^{2}-\left(\frac{W}{2}\right)^{2}} \\
H=R-\sqrt{R^{2}-\left(\frac{W}{2}\right)^{2}}
\end{gathered}
$$

熔覆层高度 $H$ 和熔覆层外轮廓半径 $R$ 之间关系 还可以简化表达成 ${ }^{[27]}$

$$
H=\frac{\Omega}{R L}
$$

式中, $\Omega$ 为丝材熔化时体积; $L$ 为单道熔覆层长度; $\Omega$ 可以表达为

$$
\Omega=\pi r^{2} V_{f}\left(\frac{L}{V_{s}}\right) \frac{\rho_{0}}{\rho_{m}}
$$

根据式(18)、(19), 求出

$$
H=\frac{\pi r^{2} V_{f} \rho_{0}}{V_{s} R \rho_{m}}
$$

根据熔覆层宽度 $W$ 、光斑直径 $d_{2}$ 以及扫描速度 $V_{s}$ 经验公式 ${ }^{[28]}$, 其中 $a$ 为经验常数

$$
W=d_{2}\left(1-a V_{s}\right)
$$

单位能量密度 $E_{1}$ 减少, 导致对流不充分, 熔池 宽度 $W$ 变小, 而光斑直径光斑 $d_{2}$ 也与熔池宽度 $W$ 相关, 光斑直径 $d_{2}$ 大, 则熔池宽度 $W$ 相应变大。 外径 $d_{2}$ 和单位能量密度 $E_{1}$ 共同决定熔覆层几何特 征, 为了能够准确预测熔覆层几何特征变化规律, 由式(15) (17) 以及(20)求得熔覆层宽度 $W_{1}$, 再由 式(15) (17)、(20)以及(21)在负离焦 $2.5 \mathrm{~mm}$ 情况求 解出经验常数 $a$, 最终通过式(21)根据光斑外径 $d_{2}$ 变化, 求解出 $W_{2}$, 取 $W_{1}$ 和 $W_{2}$ 的平均值, 作为最 终预测熔覆层宽度 $W$ 值, 计算出离焦量 $-3.0 \mathrm{~mm}$ 、 $-2.5 \mathrm{~mm}$ 以及 $-2.0 \mathrm{~mm}$ 的熔覆层宽度 $W$, 如表 5 所示。

\section{表 5 不同离焦量熔覆层宽度 $\mathrm{W}$ 预测值}

\begin{tabular}{lccc}
\hline \multicolumn{1}{c}{ 离焦量 $/ \mathrm{mm}$} & -3.0 & -2.5 & -2.0 \\
\hline 熔覆层宽度 $W_{1} / \mathrm{mm}$ & 3.6 & 3.8 & 4.3 \\
熔覆层宽度 $W_{2} / \mathrm{mm}$ & 4.5 & 3.8 & 3.0 \\
熔覆层宽度 $W\left(\left(W_{1}+W_{2}\right) / 2\right) / \mathrm{mm}$ & 4.1 & 3.8 & 3.7 \\
\hline
\end{tabular}

(2) 几何特征分析。

从表 5 预测值看出, 由于光斑直径 $d_{2}$ 变化导致 的熔覆层宽度 $W_{2}$ 变化大于单位能量密度 $E_{1}$ 所引起 $W_{1}$ 变化, 从而出现了熔覆层宽度 $W$ 随着离焦量增 大而相应增大, 由于送丝速度和扫描速度不变, 单 位长度熔覆层质量保持不变, 熔覆层宽度 $W$ 增加, 高度 $H$ 会相应减少，宽高比 $a$ 增大，与图 6 所测量 的熔覆层几何特征尺寸的变化规律基本吻合。数学 模型计算出来熔覆层几何特征值和实际测量值存在 一定的误差, 主要原因是数学模型中材料属性的数 值、激光功率吸收率是固定值, 在实际试验过程中, 
并不是恒定的, 而是随着温度变化而变化; 热量损 失主要考虑丝材熔化过程中产生对流和辐射损耗, 忽略保护气体对能量损耗的影响; 所假设的圆弧状

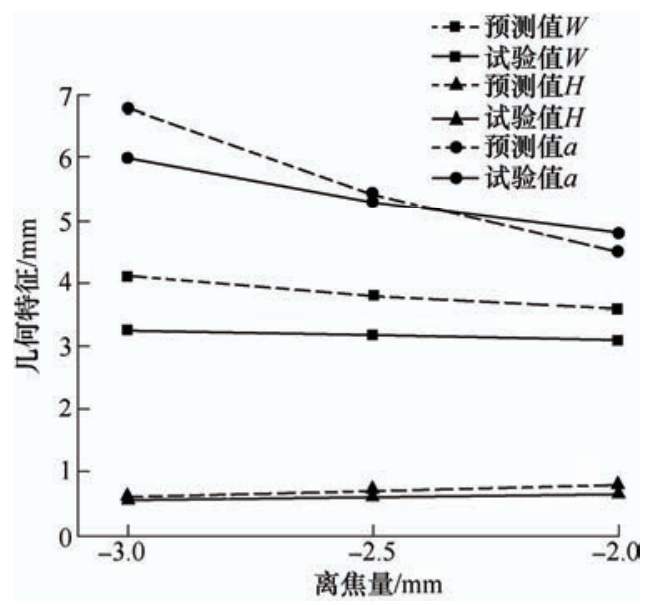

图 6 离焦量对熔覆层几何特征影响图

的熔覆层轮廓和实际熔覆层还是有一定的误差的; 数 学模型中的光斑为沿着三个光斑外径相切直径为 $d_{2}$ 实心光斑, 而实际光斑是由周向均匀分布的三个独立 光斑组成。后续激光功率、扫描速度以及送丝速度数 学模型计算值和实际测量值的主要误差原因同离焦 量, 后续不再赘述。根据试验结果, 离焦量对熔覆层 几何特征影响比较大, 选择合理的离焦量十分必要, 离焦量- $2.5 \mathrm{~mm}$ 时, 能够得到表面形貌好和宽高比大 的熔覆层, 以下试验中均采用此离焦量。

\section{2 激光功率}

\subsection{1 试验结果}

表 6 为离焦量 $-2.5 \mathrm{~mm}$, 送丝速度 $20.5 \mathrm{~mm} / \mathrm{s}$, 扫 描速度 $6 \mathrm{~mm} / \mathrm{s}$, 激光功率 $1.0 \sim 1.6 \mathrm{~kW}$ 区间变化单道 熔覆层表面形貌经历四种状态: (1) 激光功率为 $1.0 \mathrm{~kW}$ 时, 熔覆层表面呈 “残缺” 状, 局部有 “粘丝” 现象; (2) 激光功率为 $1.1 \mathrm{~kW}$ 时, 可以形成整道熔覆层, 熔 覆层局部区域有 “凹陷” 状; (3) 激光功率为 $1.2 \mathrm{~kW}$ 、 $1.3 \mathrm{~kW} 、 1.4 \mathrm{~kW}$ 和 $1.5 \mathrm{~kW}$ 时, 熔覆层表面连续光滑且 均匀; (4) 激光功率为 $1.6 \mathrm{~kW}$ 时, 熔覆层表面呈不连 续的 “熔滴” 状。激光功率为 $1.2 \sim 1.5 \mathrm{~kW}$ 区间的熔 覆层几何特征: 根据图 7 可知, 激光功率增大, 熔覆
层宽度 $W$ 增加, 高度 $H$ 减少, 宽高比 $a$ 增加。

表 6 不同激光功率单道熔覆层表面形貌

\begin{tabular}{cccc}
\hline \multirow{2}{*}{ 试样 } & \begin{tabular}{c} 
激光功率 \\
\cline { 3 - 4 }
\end{tabular} & \multicolumn{2}{c}{ 表面形貌 } \\
\hline 1 & 1.0 & 图片 & (1) \\
\hline 2 & 1.1 & 状态 \\
\hline 3 & 1.2 & $(3)$ \\
4 & 1.3 & \\
5 & 1.4 & & (3) \\
6 & 1.5 & & (3) \\
7 & 1.6 & & (3) \\
\hline
\end{tabular}

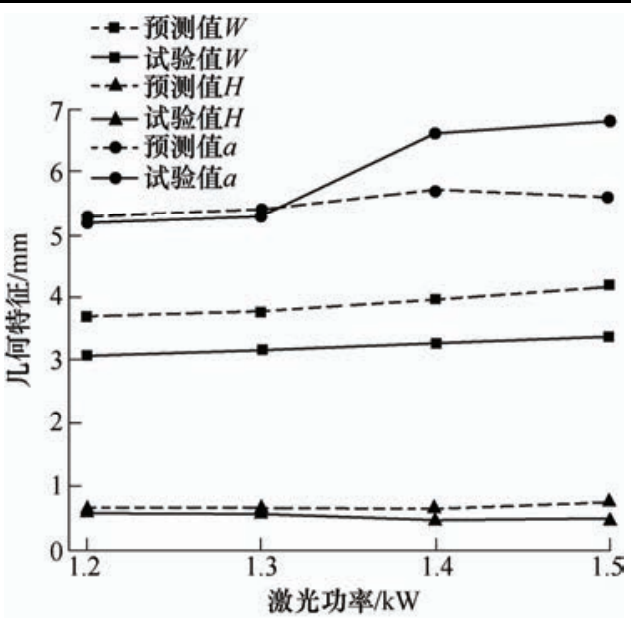

图 7 不同激光功率熔覆层几何特征图

\subsection{2 表面形貌分析}

如表 7 所示, 同理可知, 激光功率为 $1.0 \mathrm{~kW}$ 时, $E_{1}<E_{2}$, 熔池能量 “不足”, 出现熔覆层形貌(1)情况: 熔覆层表面呈 “残缺” 状, 局部有 “粘丝” 现象, 不能形成整道熔覆层, 熔覆层表面质量较差。激光 功率为 $1.1 \mathrm{~kW}$ 时, 其 $E_{1} / E_{2}$ 比值为 1.1 , 熔池能量刚 好满足熔化丝材, 熔池能量略显 “不足”, 出现熔覆 层形貌(2)情况: 可以形成整道熔覆层, 熔覆层局 部区域有 “凹陷” 状。激光功率为 $1.2 \mathrm{~kW} 、 1.3$ $\mathrm{kW} 、 1.4 \mathrm{~kW} 、 1.5 \mathrm{~kW}$ 时, $E_{1} / E_{2}$ 比值区间在 $1.2 \sim$ 1.5 之间, 熔池能量 “充足”, 获得了连续光滑且 均匀的形貌(3)。激光功率为 $1.6 \mathrm{~kW}$ 时, $E_{1} / E_{2}$ 比 值高达 1.7, 熔池能量 “过剩”, 熔覆层表面呈不 连续的 “熔滴” 状(4)。

表 7 不同激光功率单位能量密度值

\begin{tabular}{cccccccc}
\hline 激光功率 $/ \mathrm{kW}$ & 1.0 & 1.1 & 1.2 & 1.3 & 1.4 & 1.5 & 1.6 \\
\hline 能量密度 $E_{2} /\left(\mathrm{J} / \mathrm{m}^{2}\right)$ & $1.7 \times 10^{7}$ & $1.7 \times 10^{7}$ & $1.7 \times 10^{7}$ & $1.7 \times 10^{7}$ & $1.7 \times 10^{7}$ & $1.7 \times 10^{7}$ & $1.7 \times 10^{7}$ \\
能量密度 $E_{1} /\left(\mathrm{J} / \mathrm{m}^{2}\right)$ & $1.6 \times 10^{7}$ & $1.8 \times 10^{7}$ & $2.1 \times 10^{7}$ & $2.2 \times 10^{7}$ & $2.4 \times 10^{7}$ & $2.6 \times 10^{7}$ & $2.9 \times 10^{7}$ \\
$E_{1} / E_{2}$ & 0.9 & 1.1 & 1.2 & 1.3 & 1.4 & 1.5 & 1.7 \\
\hline
\end{tabular}

\subsection{3几何特征分析}

通过上述计算公式, 求出激光功率 $1.2 \mathrm{~kW}$ 、 $1.3 \mathrm{~kW} 、 1.4 \mathrm{~kW}$ 以及 $1.5 \mathrm{~kW}$ 时对应的熔覆层预测值
宽度 $W$ 、高度 $H$ 以及宽高比 $a$, 同理, 激光功率 $P$ 增加, $E_{1}$ 相应增加, 熔池表面张力越大, 熔液更加 容易对流到熔池两侧, 熔池宽度尺寸 $W$ 就会变大, 
和试验结果变化趋势基本保持一致; 而熔覆层实际 高度 $H$ 与预测值 $H$ 相比反而略有增加, 根据数学模 型式(15)可知, 激光功率 $P$ 增加, 熔覆层横截面积 $S$ 会增加, 导致宽度 $W$ 增加同时, 高度 $H$ 也会略有增 加, 而实际熔覆层高度 $H$ 却减小, 是因为此试验过 程中送丝速度 $V_{\mathrm{f}}$ 和扫描速度 $V_{\mathrm{s}}$ 保持不变, 即单位长 度熔覆层质量保持不变, 熔覆层宽度 $W$ 增加, 高度 $H$ 会相应减少, 从而导致熔覆层高度 $H$ 以及宽高比 $a$ 相比预测值变化趋势略有差异, 但总体差异非常小。

\section{3 扫描速度}

\subsection{1 试验结果}

表 8 为离焦量 $-2.5 \mathrm{~mm}$, 激光功率 $1.3 \mathrm{~kW}$, 送丝 速度 $20.5 \mathrm{~mm} / \mathrm{s}$, 扫描速度在 $3.0 \sim 9.5 \mathrm{~mm} / \mathrm{s}$ 区间单 道熔覆层表面形貌经历四种状态: (1) 扫描速度为 $3.0 \mathrm{~mm} / \mathrm{s}$ 时, 熔覆层宽度方向呈 “锯齿” 状, 熔覆 层表面有 “凹陷” 状; (2) 扫描速度为 $3.5 \mathrm{~mm} / \mathrm{s}$ 时, 熔覆层表面呈 “凹陷” 状; (3) 扫描速度为 4.5 $8.5 \mathrm{~mm} / \mathrm{s}$ 时, 熔覆层表面连续光滑且均匀; (4) 扫描 速度为 $9.5 \mathrm{~mm}$ 时, 熔覆层表面呈 “残缺” 状, 局 部有 “粘丝” 状。扫描速度为 $4.5 \sim 8.5 \mathrm{~mm} / \mathrm{s}$ 区间的 几何特征: 根据图 8 可知, 扫描速度 $V_{s}$ 增大, 熔覆 层宽度 $W$ 减少, 高度 $H$ 减少, 宽高比 $a$ 略有增加。

\section{表 8 不同扫描速度单道熔覆层形貌}

\begin{tabular}{cccc}
\hline 试样 & $\begin{array}{c}\text { 扫描速度 } \\
\text { /(mm/s) }\end{array}$ & \multicolumn{2}{c}{ 表面形貌 } \\
\hline 1 & 3.0 & 图片 & 状态 \\
\hline 2 & 3.5 & 1 \\
3 & 4.5 & & (2) \\
4 & 5.5 & & (3) \\
5 & 6.5 & & (3) \\
6 & 7.5 & & (3) \\
7 & 8.5 & & (3) \\
8 & 9.5 & & (4) \\
\hline
\end{tabular}

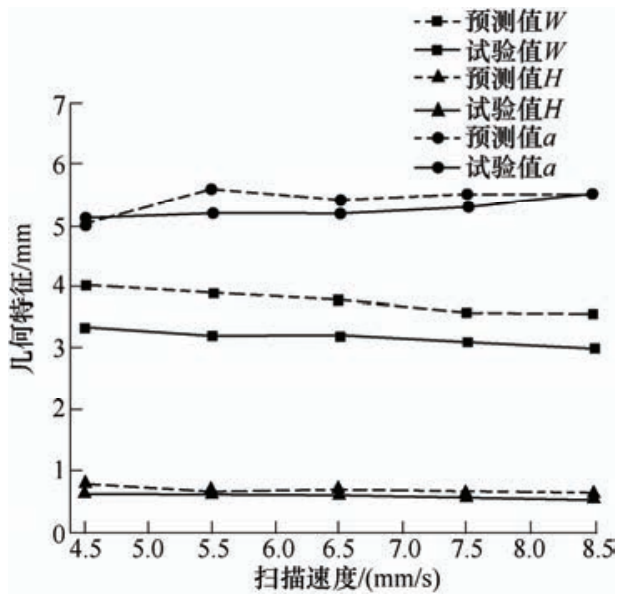

图 8 不同扫描速度熔覆层几何特征图

\subsection{2 表面形貌分析}

由表 9 可知, 扫描速度 $V_{s}$ 为 $3 \mathrm{~mm} / \mathrm{s}$ 时, $E_{1}$ 和 $E_{2}$ 都处于最大值, $E_{1}<E_{2}$, 熔池的能量 “不足”, 但 并未出现丝材未熔化的现象, 主要是因为激光所提 供的单位能量密度 $E_{1}$ 较大, 热传导的作用下会使整 个熔池面积增大, 即使丝材稍微偏离中心依然会被 熔化, 所以出现了熔覆层形貌(1)的情况：熔覆层宽 度方向呈 “锯齿” 状, 熔覆层表面有 “凹陷” 状, 导致熔覆层表面质量较差; 扫描速度为 $3.5 \mathrm{~mm} / \mathrm{s}$ 时, 从 $E_{1} / E_{2}$ 比值刚好等于 1.1 , 同理, 丝材熔化依然不 是太稳定, 导致出现熔道形貌(2)的情况：熔覆层局 部区域有 “凹陷” 状; 扫描速度为 $4.5 \mathrm{~mm} / \mathrm{s}$ 、 $5.5 \mathrm{~mm} / \mathrm{s} 、 6.5 \mathrm{~mm} / \mathrm{s} 、 7.5 \mathrm{~mm} / \mathrm{s}$ 和 $8.5 \mathrm{~mm} / \mathrm{s}$ 时, $E_{1} / E_{2}$ 比值区间在 $1.2 \sim 1.5$, 熔池能量 “充足” 完全可以 熔化进入熔池的丝材, 则可以获得了熔覆层连续光 滑且均匀的形貌(3)。扫描速度为 $9.5 \mathrm{~mm} / \mathrm{s}$ 时, 虽然 $E_{1} / E_{2}$ 比值达到 1.6, 但并未出现上述 “熔滴” 状, 而是出现了相反的状态: 丝材没有被完全熔化的“残 缺” 状(4), 是因为激光所能提供单位能量密度 $E_{1}$ 比其它扫描速度都要小, 熔池能量比较低, 再加上 扫描速度增加, 会加剧丝材的偏摆, 导致熔覆过程 不稳定, 无法形成高质量熔覆层。

表 9 不同扫描速度单位能量密度值

\begin{tabular}{ccccccccc}
\hline 扫描速度 $/(\mathrm{mm} / \mathrm{s})$ & 3.0 & 3.5 & 4.5 & 5.5 & 6.5 & 7.5 & 8.5 \\
\hline 能量密度 $E_{2} /\left(\mathrm{J} / \mathrm{m}^{2}\right)$ & $3.4 \times 10^{7}$ & $2.9 \times 10^{7}$ & $2.3 \times 10^{7}$ & $1.8 \times 10^{7}$ & $1.6 \times 10^{7}$ & $1.4 \times 10^{7}$ & $1.2 \times 10^{7}$ & $1.0 \times 10^{7}$ \\
能量密度 $E_{1} /\left(\mathrm{J} / \mathrm{m}^{2}\right)$ & $3.1 \times 10^{7}$ & $3.2 \times 10^{7}$ & $2.8 \times 10^{7}$ & $2.4 \times 10^{7}$ & $2.2 \times 10^{7}$ & $2.0 \times 10^{7}$ & $1.8 \times 10^{7}$ & $1.6 \times 10^{7}$ \\
$E_{1} / E_{2}$ & 0.9 & 1.1 & 1.2 & 1.3 & 1.4 & 1.4 & 1.5 \\
\hline
\end{tabular}

\subsection{3几何特征分析}

扫描速度 $V_{s}$ 增加, $E_{1}$ 和 $E_{2}$ 相应减小, 即熔池 能量减小, 熔池对流减弱, 以及进入熔池的丝材也 相应减少, 从而导致熔覆层宽度 $W$ 和高度 $H$ 同时减 小, 宽高比 $a$ 总体略有上升, 如图 8 所示, 和实际
试验结果变化趋势基本保持一致。

\section{4 送丝速度}

\subsection{1 试验结果}

表 10 为离焦量 $-2.5 \mathrm{~mm}$, 激光功率 $1.3 \mathrm{~kW}$, 扫描 速度 $6 \mathrm{~mm} / \mathrm{s}$, 送丝速度在 14.5 $26.5 \mathrm{~mm} / \mathrm{s}$ 区间变化 
单道熔覆层表面形貌经历五种状态: (1) 送丝速度为 $14.5 \mathrm{~mm} / \mathrm{s}$ 时, 熔覆层表面呈不连续的 “熔滴” 状; (2) 送丝速度为 $16.0 \mathrm{~mm} / \mathrm{s}$ 和 $17.5 \mathrm{~mm} / \mathrm{s}$ 时, 熔覆层表 面呈 “凹陷” 状; (3) 送丝速度为 $19.0 \sim 23.5 \mathrm{~mm} / \mathrm{s}$ 时, 熔覆层表面连续光滑且均匀; (4) 送丝速度为 $25.0 \mathrm{~mm} / \mathrm{s}$ 时, 熔覆层表面呈“山陷” 状; (5) 送丝速度为 $26.5 \mathrm{~mm} / \mathrm{s}$ 时, 呈 “粘丝” 状; 送丝速度为 $19.0 \sim 23.5 \mathrm{~mm} / \mathrm{s}$ 区间 熔覆层几何特征: 如图 9 所示, 送丝速度 $V_{f}$ 增大, 熔 覆层宽度 $W$ 和高度 $H$ 都会增大, 宽高比 $a$ 减小。

表 10 不同送丝速度单道熔覆层形貌

\begin{tabular}{|c|c|c|c|}
\hline \multirow{2}{*}{ 试样 } & 送丝速度 & \multicolumn{2}{|l|}{ 表面形貌 } \\
\hline & $/(\mathrm{mm} / \mathrm{s})$ & 图片 & 状态 \\
\hline 1 & 14.5 & $-9+2=$ & (1) \\
\hline 2 & 16.0 & 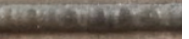 & (2) \\
\hline 3 & 17.5 & 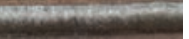 & (2) \\
\hline 4 & 19.0 & nave & (3) \\
\hline 5 & 20.5 & 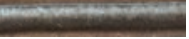 & (3) \\
\hline 6 & 22.0 & 2his & (3) \\
\hline 7 & 23.5 & & (3) \\
\hline 8 & 25.0 & 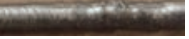 & (4) \\
\hline 9 & 26.5 & 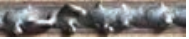 & (5) \\
\hline
\end{tabular}

\section{4 .2 表面形貌分析}

由表 11 可知, 送丝速度 $V_{s}$ 增大, 丝材熔化过程 中产生对流和辐射所带来损耗就大, 导致激光提供有 效单位能量密度 $E_{1}$ 减小, 由于进入熔池需要熔化的丝 材增多, 丝材熔化所需要的能量密度 $E_{2}$ 增加。送丝速 度 $V_{f}$ 为 $14.5 \mathrm{~mm} / \mathrm{s}$ 时, $E_{1} / E_{2}$ 的比值高达 2.1, 同理可 知, 熔池能量 “过剩”, 熔覆层表面形成不连续的 “熔 滴” 状(1); 送丝速度为 $16.0 \mathrm{~mm} / \mathrm{s}$ 和 $17.5 \mathrm{~mm} / \mathrm{s}$ 时, 熔 池能量略显 “过剩”, $E_{1} / E_{2}$ 的比值为 1.8 和 1.6, 丝材 进入熔池依然不够平稳, 熔覆层表面局部有 “凹陷” 状(2), 表面质量较差; 送丝速度为 $19.0 \mathrm{~mm} / \mathrm{s}$ 、 $20.5 \mathrm{~mm} / \mathrm{s} 、 22.0 \mathrm{~mm} / \mathrm{s}$ 和 $23.5 \mathrm{~mm} / \mathrm{s}$ 时, $E_{1} / E_{2}$ 的比值
区间为 $1.1 \sim 1.5$, 熔池能量 “充足” 可以获得的表面 形貌佳的熔覆层(3); 送丝速度为 $25.0 \mathrm{~mm} / \mathrm{s}$ 时, $E_{1} / E_{2}$ 的比值为 1 , 丝材熔化不充分, 轻微顶到基材, 搅动 熔池, 导致出现熔覆层形貌(4): 熔覆层表面呈 “凹陷” 状; 送丝速度为 $26.5 \mathrm{~mm} / \mathrm{s}$ 时, $E_{1} / E_{2}$ 比值为 0.9 , 熔 池的能量略显 “不足”, 丝材不能完全熔化, 出现了 熔覆层形貌(5)：熔覆层表面呈 “粘丝” 状。

\subsection{3 几何特征分析}

通过质量守恒定律式(14)以及图 9 可知, 扫描 速度 $V_{s}$ 不变, 送丝速度 $V_{f}$ 与熔覆层横截面积 $S$ 成正 比关系, 送丝速度 $V_{f}$ 越大, 熔覆层横截面积 $S$ 就越 大, 熔覆层宽度 $W$ 和高度 $H$ 就相应的增大, 和试验 结果变化趋势基本保持一致; 由表 11 和图 9

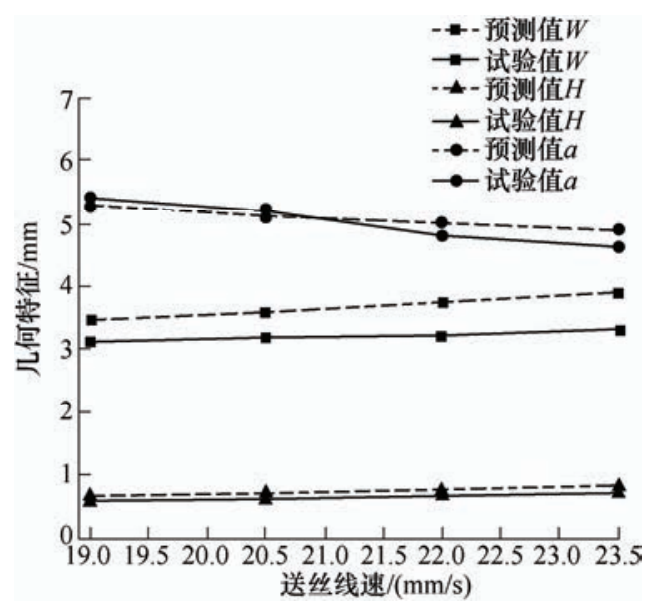

图 9 不同送丝速度熔覆层几何特征图

可知, 送丝速度为 $19.0 \sim 23.5 \mathrm{~mm} / \mathrm{s}$ 区间, 激光提供 能量密度 $E_{1}$ 变化甚小, 即熔池能量变化小, 熔池对 流差异不大, 导致熔覆层宽度尺寸 $W$ 变化甚微, 但 由于送丝速度 $V_{f}$ 使得熔覆层横截面积 $S$ 增大，使得 多出来的熔覆层质量只能在纵向方向累积, 从而导 致熔覆层高度尺寸 $H$ 明显增大, 宽高比 $a$ 由大变小。

表 11 不同送丝速度单位能量密度值

\begin{tabular}{cccccccccc}
\hline 送丝速度/(mm/s) & 14.5 & 16.0 & 17.5 & 19.0 & 20.5 & 22.0 & 23.5 & 25.0 & 26.5 \\
\hline$E_{2} /\left(\mathrm{J} / \mathrm{m}^{2}\right)$ & $1.2 \times 10^{7}$ & $1.3 \times 10^{7}$ & $1.4 \times 10^{7}$ & $1.5 \times 10^{7}$ & $1.7 \times 10^{7}$ & $1.8 \times 10^{7}$ & $1.9 \times 10^{7}$ & $2.1 \times 10^{7}$ & $2.1 \times 10^{7}$ \\
$E_{1} /\left(\mathrm{J} / \mathrm{m}^{2}\right)$ & $2.5 \times 10^{7}$ & $2.4 \times 10^{7}$ & $2.3 \times 10^{7}$ & $2.3 \times 10^{7}$ & $2.2 \times 10^{7}$ & $2.2 \times 10^{7}$ & $2.1 \times 10^{7}$ & $2.1 \times 10^{7}$ & $2.0 \times 10^{7}$ \\
$E_{1} / E_{2}$ & 2.1 & 1.8 & 1.6 & 1.5 & 1.3 & 1.2 & 1.1 & 1.0 & 0.9 \\
\hline
\end{tabular}

\section{3 结论}

(1) 建立了三光束激光熔覆工艺参数与熔覆层 表面形貌关系的数学模型: 通过激光提供的单位能 量密度 $E_{1}$ 与丝材所需能量密度 $E_{2}$ 之间比值关系判 断熔覆层表面形貌状态, 并采用单因素试验法展开 验证, 结果表明: (1) 激光提供的单位能量密度 $E_{1}$
小于丝材所需能量密度 $E_{2}$, 即 $E_{1} / E_{2}<1$, 熔池能量 不足, 熔覆层表面主要呈 “残缺” 状, 局部有 “粘 丝” 现象或熔覆层宽度方向呈 “锯齿” 状, 熔覆层 表面有 “凹陷” 状; (2) 激光提供的单位能量密度 $E_{1}$ 大于等于丝材所需能量密度 $E_{2}$, 且 $1.1 \geqslant E_{1} / E_{2} \geqslant$ 1.0 , 熔池能量处于 “不足” 到 “充足” 过渡阶段, 丝材熔化不稳定，熔覆层局部区域有 “凹陷” 状;

(3) 激光提供的单位能量密度 $E_{1}$ 大于丝材所需能量 
密度 $E_{2}$, 且 $1.5 \geqslant E_{1} / E_{2}>1.1$, 熔池能量 “充足” 能 够充分熔化进入熔池的丝材, 以及丝材能够以连续平 稳的 “搭桥过渡” 方式熔入熔池, 熔覆层表面连续光 滑, 质量较高; (4) 激光提供的单位能量密度 $E_{1}$ 远远 大于丝材所需能量密度 $E_{2}$, 且 $E_{1} / E_{2}>1.6$, 熔池能量 “过 剩” , 熔覆层表面主要呈 “熔滴” 状或 “凹陷” 状。

(2) 通过工艺参数和熔覆层几何特征关系的数 学模型计算得出熔覆层宽度 $W$ 、高度 $H$ 以及宽高比 $a$ 变化趋势基本和试验结果吻合: 激光功率 $1.3 \mathrm{~kW}$, 送丝速度 $20.5 \mathrm{~mm} / \mathrm{s}$, 扫描速度 $6 \mathrm{~mm} / \mathrm{s}$, 离焦量从 $-3 \mathrm{~mm}$ 减小到 $-2 \mathrm{~mm}$ 过程中, 熔覆层宽度 $W$ 减少, 高度 $H$ 增大, 宽高比 $a$ 减少; 离焦量 $-2.5 \mathrm{~mm}$, 送 丝速度 $20.5 \mathrm{~mm} / \mathrm{s}$, 扫描速度 $6 \mathrm{~mm} / \mathrm{s}$, 激光功率从 $1.2 \mathrm{~kW}$ 增加到 $1.5 \mathrm{~kW}$ 过程中, 熔覆层宽度 $W$ 增加, 高度 $H$ 减小, 宽高比 $a$ 增加。离焦量 $-2.5 \mathrm{~mm}$, 激 光功率 $1.3 \mathrm{~kW}$, 送丝速度 $20.5 \mathrm{~mm} / \mathrm{s}$, 扫描速度从 $4.5 \mathrm{~mm} / \mathrm{s}$ 增加到 $8.5 \mathrm{~mm} / \mathrm{s}$, 熔覆层宽度 $W$ 减少, 高 度 $H$ 减少, 宽高比 $a$ 略有增加。离焦量 $-2.5 \mathrm{~mm}$, 激光功率 $1.3 \mathrm{~kW}$ ，扫描速度 $6 \mathrm{~mm} / \mathrm{s}$, 送丝速度从 $19.0 \mathrm{~mm} / \mathrm{s}$ 增加到 $23.5 \mathrm{~mm} / \mathrm{s}$, 送丝速度增大, 熔覆 层宽度 $W$ 和高度 $H$ 都会增大，宽高比 $a$ 减小。

\section{参 考 文 献}

[1] 董世运，马运哲，徐滨士，等. 激光熔覆材料研究现状 [J]. 材料导报，2006，20(6): 5-9.

DONG Shiyun, MA Yunzhe, XU Binshi, et al. Current status of material for laser cladding [J]. Material Review, 2006, 20(6): 5-9.

[2] SYED W H H, LI Lin. Effects of wire feeding direction and location in multiple layer diode laser direct metal deposition[J]. Applied Surface Science, 2005, 248: 518-524.

[3] 石世宏, 傅戈雁, 李龙, 等. 中空激光光内同轴送丝熔 覆工艺的实现及其试验研究 $[\mathrm{J}]$. 中国激光, 2010, 37(1): 266-270.

SHI Shihong, FU Geyan, LI Long, et al. Realization and research of laser cladding with method of internal wire feeding through a hollow laser beam[J]. Chinese Journal of Laser, 2010， 37(1): 266-270.

[4] 朱刚贤, 石拓, 傅戈雁, 等. 中空光光内送丝熔覆工艺 参数对熔覆层质量的影响 $[\mathrm{J}]$. 应用激光, 2013, 33(4): 381-384.

ZHU Gangxian, SHI Tuo, FU Geyan, et al. Effect of process parameters on the quality of the cladding layer by the inside-laser wire feeding[J]. Applied Laser, 2013, 33(4): 381-384.

[5] LIU Shuang, LIU Wei, HAROONI M, et al. Real-time monitoring of laser hot-wire cladding of Inconel 625[J]. Optics and Laser Technology, 2014, 62: 124-134.

[6] SHI Jianjun, ZHU Ping, FU Geyan, et al. Geometry characteristics modeling and process optimization in coaxial laser inside wire cladding[J]. Optics and Laser Technology, 2018, 101: 341-348.

[7] 傅戈雁, 吉绍山, 石世宏, 等. 一种用于激光熔覆的激 光光内送丝装置：CN201610075735[P]. 2017-09-05.

FU Geyan，JI Shaoshao，SHI Shihong, et al. A device of coaxial wire feeding through beams for laser cladding: CN201610075735[P]. 2017-09-05.

[8] 傅戈雁, 吉绍山, 刘凡, 等. 激光熔覆装置的抛物面的 设定方法及激光熔覆装置：CN201710662057.1[P]. 2019-04-12.

FU Geyan, JI Shaoshao, LIU Fan, et al. Setting method of parabola of laser cladding device and laser cladding device: CN201710662057.1[P]. 2019-04-12.

[9] 傅戈雁, 吉绍山, 刘凡, 等.激光熔覆装置 : CN201710661517.9[P]. 2017-09-29.

FU Geyan, JI Shaoshao, LIU Fan, et al. Laser cladding device: CN201710661517.9[P]. 2017-09-29.

[10] 傅戈雁，吉绍山，刘凡，等.激光熔覆装置： CN201710661537.6[P]. 2017-10-03.

FU Geyan, JI Shaoshao, LIU Fan, et al. Laser cladding device: CN201710661537.6[P]. 2017-10-03.

[11] 李凯斌, 李东, 刘东宇, 等. 光纤激光送丝熔覆修复工 艺研究[J]. 中国激光，2014，41(11): 76-81.

LI Kaibin, LI Dong, LIU Dongyu, et al. Research of fiber laser cladding repairing process with wire feeding[J]. Chinese Journal of Laser, 2014， 41(11): 76-81.

[12] 罗园青. 基于送丝熔敷的激光成形技术研究[D]. 武汉: 华中科技大学, 2016.

LUO Yuanqing. Study on laser wire-melting deposition technology[D]. Wuhan: Huazhong University of Science and Technology, 2016.

[13] MOK S, BI G, FOLKES J, et al. Deposition of Ti-6Al-4V using a higher power diode laser and wire, partI : Investigation on the process characteristics[J]. Surface \& Coating Technology, 2008, 202: 3933-3939.

[14] ABIOYE TE, FOLKES J, CLARE A T. A parametric study of inconel 625 wire laser deposition[J]. Journal of Material Processing Technology, 2013, 213: 2145-2151.

[15] LIU Shuang, LIU Wei, KOVACEVIC R. Experimental investigation of laser hot-wire cladding [J]. Institution of Mechanical Engineers, 2015: 1-14.

[16] BARROI A, GONCALVES D, HERMSDORF J, et al. Influence of laser power on the shape of single tracks in scanner based laser wire cladding[J]. Physics Procedia., 2016: 667-673. 
[17] WEN Peng, SHAN Jiguo, ZHENG Shiqing, et al. Control of wire transfer behaviors in hot wire laser welding[J]. Int. J. Adv. Manuf. Technol., 2015: 1-10.

[18] 吉绍山, 刘凡, 傅戈雁, 等. 三光束光内同轴送丝激光 熔覆成形新方法研究 $[\mathrm{J}]$. 表面技术, 2019，48(4): 287-289.

JI Shaoshan, LIU Fan, FU Geyan, et al. New forming method of coaxial wire feeding inside three beams laser cladding [J]. Surface Technology, 2019, 48(4): 287-289.

[19] 雷定中, 石世宏, 傅戈雁. 宽带激光内送粉熔覆工艺研 究[J]. 中国激光, 2015, 42(11): 1-8.

LEI Dingzhong, SHI Shihong, FU Geyan. Research of hollow broadband laser cladding method $[\mathrm{J}]$. Chinese Journal of Laser， 2015， 42(11): 1-8.

[20] 曹明翠, 郑启光. 激光热加工[M]. 武汉: 华中工学院 出版社, 1995.

CAO Mingcui, ZHENG Qiguang. Laser hot working[M]. Wuhan: Huazhong Institute of Technology Press, 1995.

[21] 吴宗凡, 柳美琳, 张绍举. 红外与微光技术 [M]. 北京: 国防工业出版社, 1998.

WU Zongfan, LIU Meilin, ZHANG Shaoju. Infrared and low light technology[M]. Beijing: National Defense Industry Press, 1998.

[22] 黄伟. 浴扇发动机排气系统红外特征计算与抑制技术 研究[D]. 南京: 南京航空航天大学, 2010.

HUANG Wei. Investigation of numerical calculation of IR characteristics and stealth technology of turbofan engine exhaust system[D]. Nanjing: Nanjing University of Aeronautics and Astronautics, 2010.

[23] VASINONTA A, BEUTH J L, GRIFFITH M L. A process map for consistent build conditions in the solid freeform fabrication of thin-walled structures[J]. Journal of Manufacturing Science \& Engineering, 2001, 123(4): 615-622.

[24] SUAREZ A, AMADO J, TOBAR M, et al. Study of residual stresses generated inside laser cladded plates using FEM and diffraction of synchrotron radiation[J]. Surf. Coat. Tech., 2010, 204(12-13): 1983-1988.

[25] 刘建强. 304 不锈钢激光焊接的建模与仿真[D]. 天津: 天津大学, 2004.

LIU Jianqiang. Modeling and simulating of laser welding for stainless steel 304[D]. Tianjin: Tianjin University, 2004.

[26] 高燕. 不锈钢激光焊接热源及过程的模拟与仿真 $[D]$. 天 津: 天津大学, 2006.

GAO Yan. The simulation of heat source and process in laser welding for stainless steel[D]. Tianjin: Tianjin University, 2006.

[27] PAUL S, SINGH R, YAN W. Thermal model foradditive restoration of mold steels using crucible steel[J]. Journal of Manufacturing Processes, 2016, 24: 346-354.

[28] STEEN WM, WEERASINGHE VM, MONSON P. Some aspects of the formation of laser clad tracks[J]. SPIE, 1986, 650: 226-234.

作者简介: 刘凡, 女, 1984 年出生, 博士研究生。主要从事激光增材制 造方面研究。

E-mail: liufan2005@126.com; 154647304@qq.com

傅戈雁(通信作者), 女, 1956 年出生, 博士, 教授, 博士研究生导师。 主要从事激光加工与激光增材制造方面的研究。

E-mail: fugeyan@suda.edu.cn 